\title{
Final overview of COST Action TU1406 - Quality Control of Existing Bridges
}

José C. Matos

COST Action TU1406 Chairman, University of Minho, Guimarães, Portugal

Joan R. Casas

UPCatalunya-BarcelonaTech, Barcelona, Spain

Sérgio Fernandes

ANSER Lda., Santo Tirso, Portugal

Contacting author: jmatos@civil.uminho.pt

\section{Abstract}

Across Europe, the need to manage roadway bridges efficiently led to the development of multiple management systems. Despite presenting similar system frameworks, the condition assessment procedure is one of the difference that distinguishes them. This dissimilarity constitutes a divergent mechanism that has direct interference in the decision making process leading to considerable variations in roadway bridges quality. COST Action TU1406 aims to institute a standardized roadway bridges condition assessment procedure. Such purpose requires the establishment of recommendations for the quantification of performance indicators, the definition of performance goals and a guideline for the standardization of quality control plans for bridges. By developing new approaches to quantify and assess bridge performance, as well as quality specifications to assure expected performance levels, bridge management strategies will be significantly improved, enhancing asset management of ageing structures in Europe. The work developed and achieved by COST Action TU1406 will be presented.

Keywords: roadway; bridges; performance; indicators; goals; quality; control, standardization.

\section{Introduction}

Significant worldwide research has been developed over the past years regarding the condition assessment of roadway bridges. As a result, there are nowadays several ways to assess a bridge condition.

More recently, the concept of performance indicators (PI) was introduced [1], simplifying the communication between stakeholders. However, large deviations are still verified on how these indicators are obtained. Therefore, the standardization of these procedures is very much needed.
Such normalization can be achieved through the implementation of quality control (QC) plans which compare assessed Pls with pre-specified performance goals (PG). However, these goals are even more difficult to establish as they are highly subjective, leading to a high level of QC plans dispersion.

In this context, COST Action TU1406 arose with the ambition to develop a European guideline for the establishment of QC plans for roadway bridges dealing with recent developments on bridge safety, maintenance and management, according to a lifecycle outlook. 
This guideline will focus on bridge maintenance and lifecycle performance at two levels: (i) performance indicators, (ii) performance goals. By developing new approaches to quantify and assess bridge performance, as well as quality specifications to assure an expected performance level, bridge management strategies will be significantly improved, enhancing asset management of ageing structures in Europe. The implementation of asset management should increase the integration of network and bridge performance requirements.

The first focus of TU1406 was the characterization of bridge PIs and the development of a database with operational and research PIs. The second step concerned the definition of standardized PGs and the establishment of utility functions in order to compute key performance indicators (KPI) and the development of RAMS-SHEEP (see section 3) plots. These two phases will allow the definition of a guideline for establishing QC plans in roadway bridges with emphasis in advanced deterioration predictive models.

\section{Performance Indicators}

Constructions, during their life cycle, face deterioration depending on factors such as environmental condition, natural aging, material quality and planned maintenance. Therefore, PIs for the present and future structural conditions on deterministic and probabilistic level have to be defined and determined.
Management systems, capturing different degradation processes, are very often used in relation to lifecycle analyses methods. Such systems, developed for a structural condition assessment, are usually based on deterministic performance prediction models which describe the future condition by a functional correlation between structural condition attributes, such as the structural age, and the mechanical, chemical and thermal loading processes.

PIs in particular KPIs make it possible to define a set of objectives aimed to establish QC plans which ensure desired bridge quality service. However, such plans vary from country to country.

The practical implementation of the above mentioned models requires detailed information about its variables. Therefore, it is extremely important to analyse such indicators in terms of used assessment frameworks, and in terms of the quantification procedure itself.

With this in mind, Work Group 1 (WG1) activities focused on the development of a PIs database. Preliminary works consisted in the systematic screening of practical national inspection and evaluation documents (e.g. inspection, evaluation, research etc.) in order to obtain consistent and conclusive information associated with PIs, PGs and performance thresholds (PTs) from each COST partner country. The core of the survey process for obtaining PIs and corresponding KPIs is given in Figure 1.

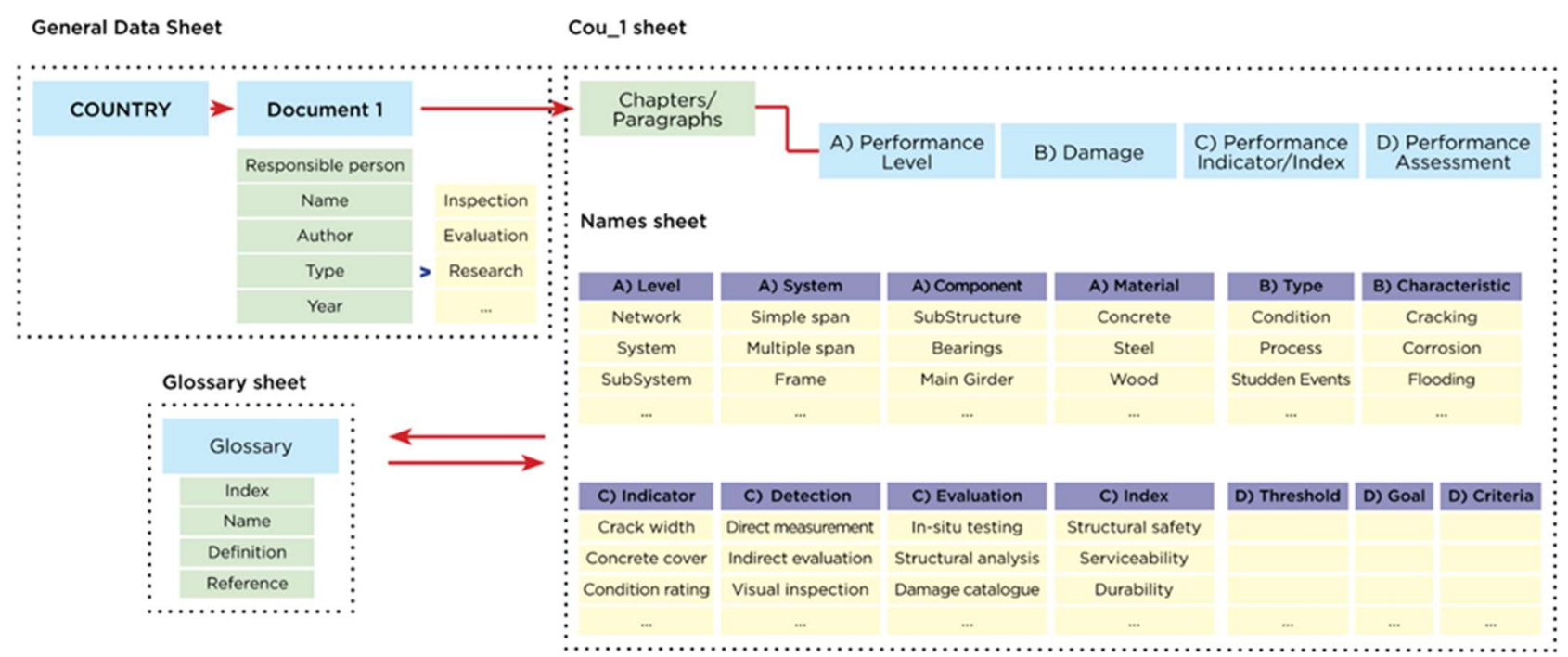

Figure 1. Structure of the performance indicators database for the survey process 
The data collection process, involving a total of 36 countries, resulted in a list of more than 700 terms considered related to PIs which was later clustered and homogenized. The screening results were then used by the core group of WG1 for a homogenization process and the nominated persons from each country were asked again to verify their performance and damage specific inputs, by comparing them with the homogenized and categorized terms. The homogenized data was the baseline for further studies and quantifications and provides also the basis for an effective comparison of performance quantities between countries.

From this process resulted a shorter list of 385 terms, grouped in 11 clusters, from defects to rating and loads. The list was considered, not as a final list of PIs, but as a list of terms that can be related to PIs (PI related terms as addressed in WG1 Report).

Assuming that not all 385 terms can be considered PIs, as such high number would make the Action aim unattainable, a new terms categorization was performed. The following four categories were considered: performance indicators (PIs), damage processes (DPs), observations (OBS) and other data (OD). A fifth category was considered, related with non-interceptable processes, for terms considered out of scope of the previous four. The sixth considered category labelled as Combined Performance Indicator clustered terms that reflect general properties of a structure and may be derived, among other ways, from the combination of several PIs. As an example, the term bridge condition was considered in this category since one can assume that it can be derived from all the other PIs. The distribution of the $385 \mathrm{PIs}$ related terms through the different categories is shown in Table 1. The complete categorization of terms can be found in the COST TU1406 WG1 report.

In order to proceed with the reduction of this list, an expert group was asked to specify PIs according to the following points: Measurable? Quantifiable? Target value available? Valid for ranking? Allow decision with economic implications? These questions are based on the definition of $\mathrm{PI}$ agreed within TU1406: performance indicator is a measurable and quantifiable parameter, related to the bridge performance, that can be directly compared with a target measure of a PG (absolute measure of performance) or can be used for ranking purposes, among a bridge population (relative measure of performance), in the framework of a Quality Control Plan or life-cycle management (decisions and actions involving economic resources).

Table 1. Distribution of the $385 \mathrm{PI}$ related terms through 8 different categories.

\begin{tabular}{lc}
\hline \multicolumn{1}{c}{ Categories } & $\begin{array}{c}\text { Number of PI } \\
\text { related Terms }\end{array}$ \\
\hline Performance Indicator & 17 \\
\hline Performance Indicator-Redundant & 7 \\
\hline Damage Process & 27 \\
\hline Damage Process - Redundant & 13 \\
\hline Observation & 245 \\
\hline Other Data & 33 \\
\hline Non-Interceptable Processes & 16 \\
\hline Combined PI Total & 27 \\
\hline \multicolumn{2}{c}{$\quad \mathbf{3 8 5}$}
\end{tabular}

\section{Performance Goals}

The concept of efficient transport network management has been introduced in the past two decades as the "process of maintaining and improving the existing road network to enable its continued use by traffic efficiently and safely, normally in a manner that is effective and environmentally sensitive; a process that is attempting to optimize the overall performance of the road network over time" [2]. Maintaining the national road network plays an important role in achieving the strategic goals. However, the relationship between goals and network performance is not exclusive. For example, network performance may be dependent on traffic patterns, weather conditions, economic growth and oil prices. It is therefore often not possible to derive PIs directly from network level goals.

Structures like bridges are necessary for a functioning transport infrastructure network. Bridge PGs can be set in order to ensure bridge performance is in line with network level PGs. When defining bridge PIs, some difficulties may present themselves. First, the timescale for which network PGs are set is typically much shorter than the estimated bridge service life. Therefore bridge 
PGs should not only enable meeting the short term PGs, but also facilitate lifecycle optimization.

Furthermore, where bridge management is traditionally focused on evaluating the bridge condition, the desired condition now needs to be expressed or translated into goals reflecting network performance.

Based on the work approach developed by Rijkswaterstaat in the Netherlands [3], a set of PIs for structures can be defined. These indicators are based on a set of performance criteria, which we refer to in our approach as performance aspects, the RAMS SHEEP criteria - acronyms for Reliability, Availability, Maintainability, Safety and Security, Health, Environment, Economic, Politics, respectively - where risk is used to obtain social, environmental, economic and political indicators. Each criterion is defined as:

- Reliability: the probability that the required function of the system can be carried out under the given conditions for a given time interval.

- Availability: the probability that the required function of the system can be carried out under the given circumstances during a given arbitrary time.

- Maintainability: the probability that the maintenance activities are possible within the specified time and under circumstances that the required function continues to run.

- Safety: related to the freedom from unacceptable risks in terms of injury to people.

- Security: related to the safety of a system regarding vandalism and unreasonable human behaviour.
- Health: being related to physically, mentally and socially defined aspects.

- Environment: concerns the physical environment requirements.

- Economics: regarding the relationship between cost and value.

- Politics: concerning political-administrative and social requirements.

A clear definition of the required performance forms the start of the process of risk based inspections. The demands on a network level will be translated to demands for parts of the network and subsequently to demands for objects, in this case bridges. Within COST Action TU1406 the terminology criteria would be related to the performance aspect and a sub-criteria would be PG. These criteria can be applied during inspection and maintenance processes [4] [5], but the link from PIs obtained and the exact criteria addressed, doesn't necessarily have to be direct.

In order to develop maintenance strategies it is necessary to establish a link between performance aspects at the bridge component level and those at the network level. The framework which links PIs and PGs at different levels is shown in Figure 2 and is based on WG1, Work Group 2 (WG2) and Work Group 1 (WG3) objectives.

However, to assess PGs, KPIs need to be defined at strategic and tactical level. Those KPIs are determined from a number of Pls collected at an operational level.

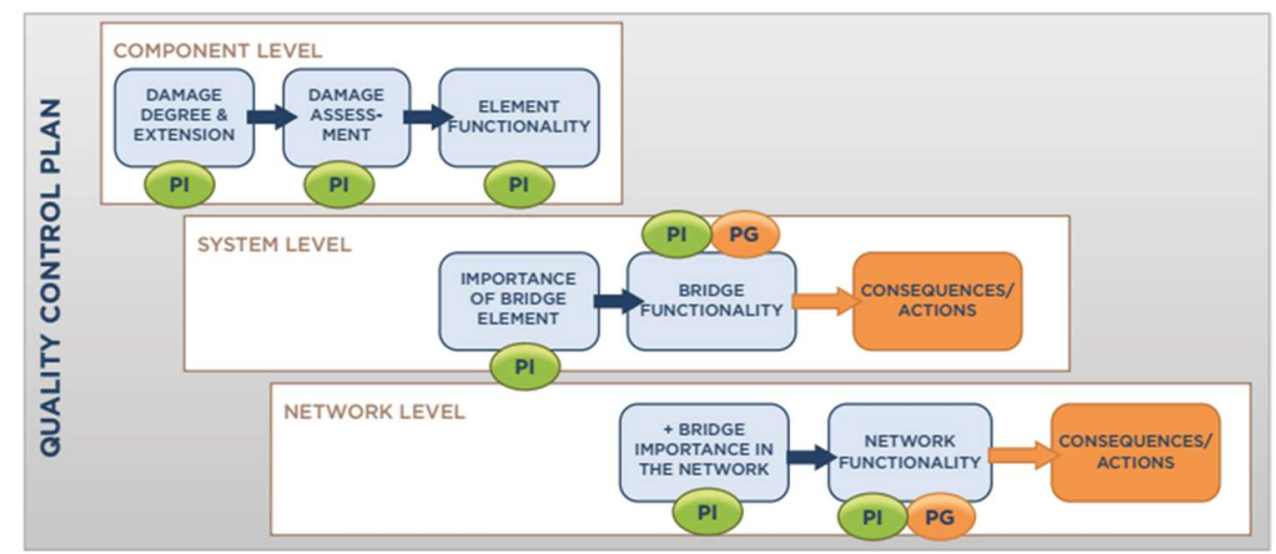

Figure 2. Assessment procedure from component to the system and network level based on the PIs and PGs 
Bridge inspections are generally carried out on the bridge component level, as shown in Figure 2, and often are divided into subsystem groups, i.e. substructure, superstructure, roadway. Through bridge inspections a number of Pls are collected and analysed (processed), in order to determine the aggregated KPI at the system level. Those PIs are usually related to technical aspects of the bridge performance, mostly defined as structural performance or reliability.

From the screening process approximately 100 extricated Pls were further related with one or more of the KPIs defined in WG2: reliability (R), availability $(A)$, maintainability $(M)$, safety $(S)$, security $(\mathrm{Se})$, health $(H)$, environment $(E)$, costs $(C)$, politics $(\mathrm{P})$, rating/inspection (I). A further process was required for categorization of PIs in relation to PGs and PTs at different levels: component $(\mathrm{CL})$, system (SL), network (NL); taking into account different aspects: technical (Tech), sustainability (Sust) and socio-economic (SoEc).

When the reliability level is in a deteriorated state, maintenance options need to be considered for each bridge below the threshold. Usually three main options are considered: (i) do nothing; (ii) do minor repair; (iii) do major repair or reconstruction.
The chosen maintenance option will have both direct and indirect impacts, such as direct costs related to the maintenance activity and indirect costs caused by maintenance activities borne by society (e.g. user delay, environmental impacts). The direct impacts are regularly determined as owner costs and will represent economy performance aspects of the bridge. Other impacts can be categorized as availability and environmental aspects. Traffic safety is also a performance aspect which can be quantified at two periods, during the regular operation and during maintenance activities.

From this intricacy between inputs can be concluded that multiple bridge PGs should be set as a multi-objective system, taking into account different aspects of bridge and network performance.

Multi-criteria decision-making (MCDM) provides a systematic approach to combine these inputs with cost/benefit information and decision-maker or stakeholder views to rank alternatives. Hierarchy structure for linking multi-objective bridge PGs, covering most of the previously mentioned aspects with PIs is shown in Figure 3.

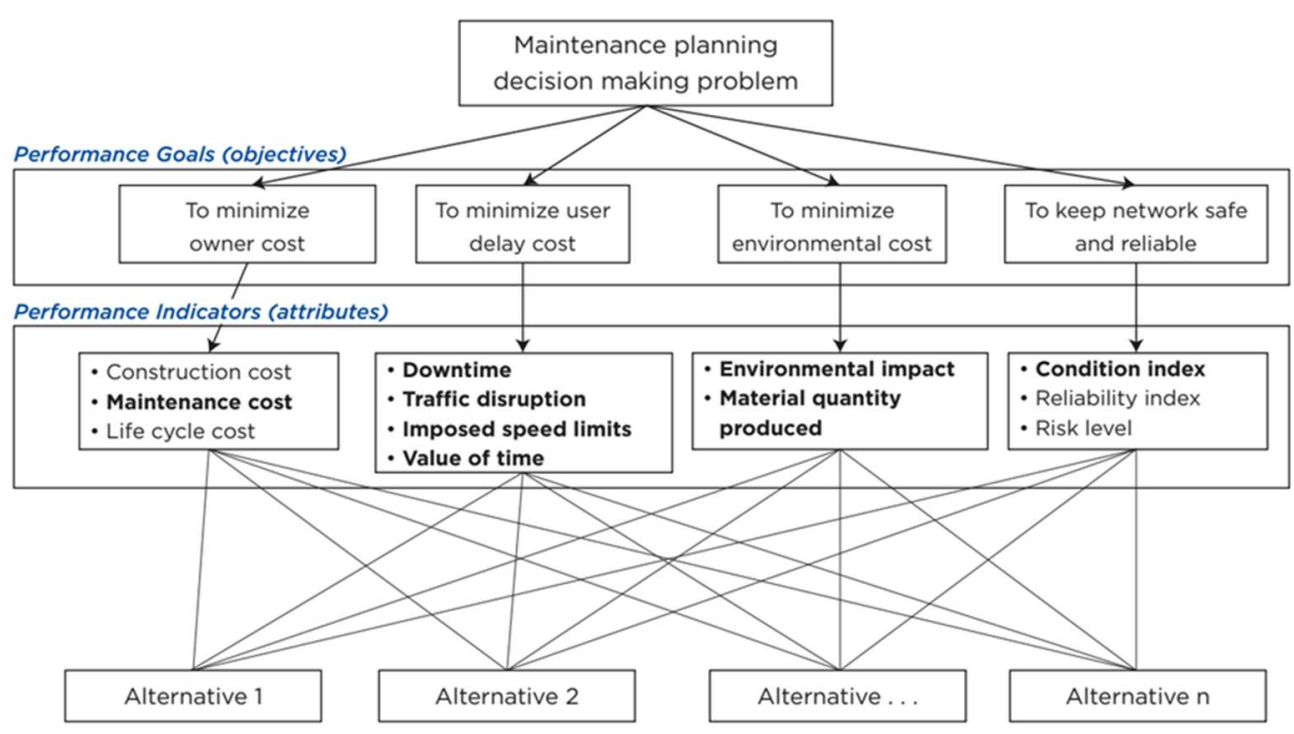

Figure 3. Linking multiple PGs (called multiple objective) to PIs (adapted from [6])

MCDM is used to identify and quantify decisionmaker and stakeholder considerations about various (mostly) non-monetary factors in order to compare alternative courses of action. Hierarchy structure for linking multi-objective bridge PGs, covering most of the previously mentioned aspects with PIs is required. Possible result of multi-criteria assessment of different bridge maintenance 
alternatives can be depicted in a spider diagram to be used for a decision making about the optimal maintenance or design solution. Alternatively, the multiple performance criteria can be combined into a so-called utility function, in which all the criteria are brought into a single scale. In order to transform the various out into a single scale (mostly monetary) it is necessary to establish weight factors for the individual types of criteria. Some of these factors are available in some countries (for example weight factor for traffic delays, noise, injuries etc.). However, depending on the selection of criteria, some weight factors may still need to be developed. In the development of the weight factors the starting point can be taken in the qualitative approach from which the apparent relative weight can be deducted.

Once the possible outcomes have been brought to a single scale, the best decision can be found as a formal optimized decision process, in which option with the maximum "utility" shall be selected as the recommended decision.

Utility theory provides a measure of preferences of a decision maker over a group of alternatives [6]. Based on the six axioms of utility theory, Multiattribute utility theory (MAUT) is introduced by [8].

MAUT provides a systematic approach to reduce the qualitative values of various attributes (i.e. PIs) into utility functions. The obtained utility scores are then aggregated based on the relative importance of attributes. The final score assigns a ranking to each alternative based on either minimization or maximization function. In other words, MAUT assigns the relative importance of PIs (e.g. condition, cost, etc.), while comparing number of bridges. These bridges are often referred as alternatives in MAUT.

MAUT involves the single decision maker who is willing to make certain trade-off among PGs while exposed with uncertainty and risk [9]. The uncertainty is usually originated due to unavailable and dynamic nature of data, and involvement of number of stakeholders. For instance, in the bridge planning the exact estimation on number of users affected due to maintenance activity is difficult to define. MAUT integrates a body of mathematical utility models and a range of decision assessment methods in order to assist in decision ranking problems [10]. The single attribute utility function is calculated for each performance aspect, which reflects the risk attitude of the decision maker.

\section{Quality Control Plan}

The quality specification or QC framework aims to provide a methodology with detailed step-by-step explanations for the establishment of QC plans for different bridge types. These plans relate user/society goals, such as:

- Reliability: including the probability of structural failure (structural safety) or operational failure (serviceability);

- Availability: the proportion of time a system is in a functioning condition. In our case is the additional travel time due to imposed traffic regime on the bridge;

- Safety (not structural safety): minimize or eliminate people harm during the service life;

- Economy: minimize life-cycle cost

- Environment: minimize the harm to environment during the service life of a bridge.

In figure 4 is shown the structure that supports the QC plan from bridges.

Quality control plans are also divided in 2 groups:

- Static (snap shot) control: to inspect and investigate the bridge and determine whether reliability (structural safety and serviceability) and safety are met. This is fundamentally the basis for the decision making on actions;

- Dynamic control: based on the static control and including the plan and actions to execute in order to ensure the long term fulfilment of safety and serviceability goals. The goals to achieve are related to availability, economy and sustainability as it includes the feasible maintenance scenarios that define costs and availability over a certain time frame by using reliability and safety forecasts.

Because the reliability goal should be checked against feasible failures, the practical application of the method has divided the global group of bridges into the following bridge types: frame, arch, continuous beam. For each of these bridge types the most vulnerable zones can be identified and the corresponding $\mathrm{PIs}$ related to reliability 
observed and/or quantified. This division also helps in the process of selecting the best maintenance policy. The goals of availability, economics and sustainability are governed by maintenance scenarios. In fact, the snapshot assessment of availability and costs are of none or little interest. Therefore, the feasible maintenance scenarios (do nothing, preventive and corrective) are defined. Regarding the availability indicator, each maintenance intervention requires certain traffic regime, which may include closure for certain type of vehicles or lane closure or narrower lanes. The normal traffic regime can be assigned with the maximum performance value. The other traffic regimes can be ranked by the additional travel time they cause for the road users. This additional travel time can be also monetized according to the guidelines given in the WG2 report [11]. The selected intervention scenario is obtained based on the MAUT.

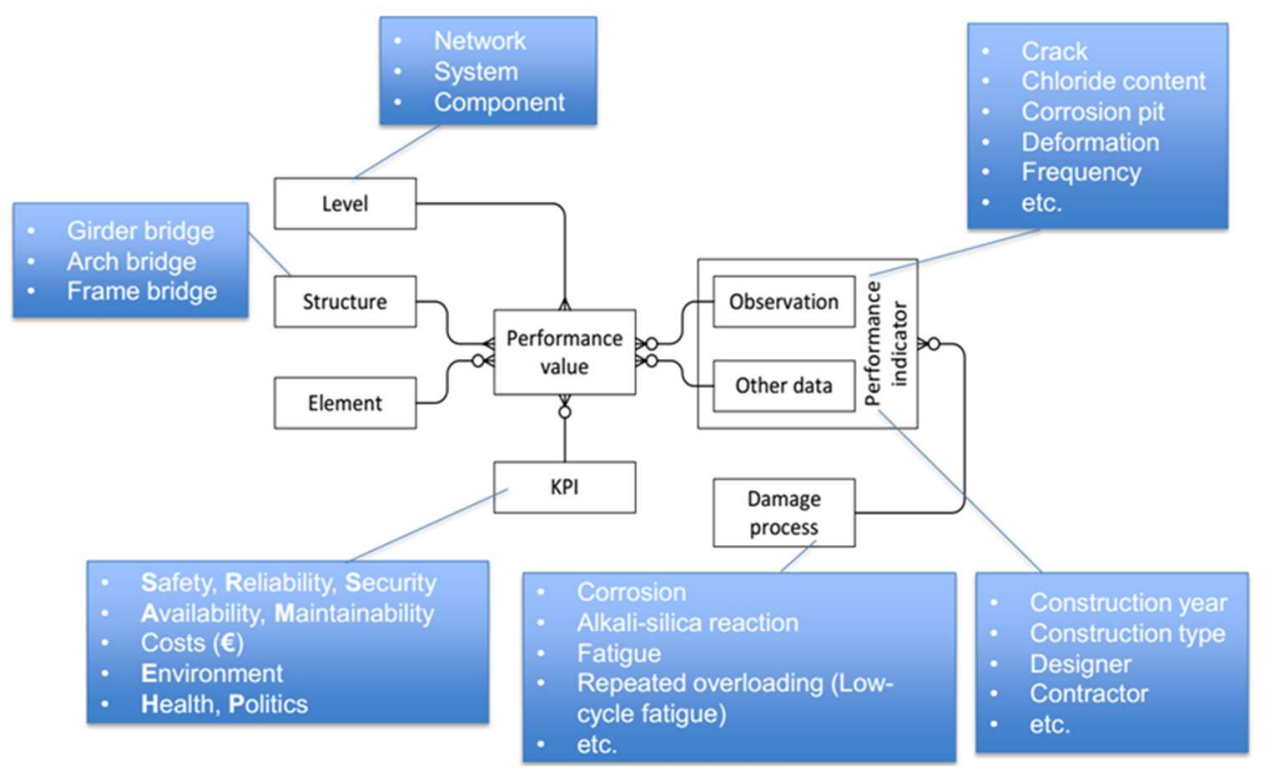

Figure 4. Quality control plan structure

\section{Conclusions}

After an exhaustive analysis of the data obtained in the research carried out, it was concluded that different countries have different definitions of PIs and how they are obtained. Due to the existence of different interpretations, an additional clustering and homogenization process was required. From this procedure it was possible to verify that all countries have a $\mathrm{PI}$, named condition index, condition rating or deterioration index, mainly obtained through visual inspections. Although in some cases this is the only existing PI used, there are countries, like Denmark or The Netherlands, in which operators and bridge owners are currently using other relevant indicators. In Denmark, concepts like remaining service life, robustness, safety index, reliability and vulnerability are addressed. On the other hand, in The Netherlands, performance is evaluated by the RAMSSHEEP approach.

A methodology to collect PIs used across Europe was presented. The aim was to establish good practices on the definition of QC plans for roadway bridges. This should be the basis of the future proposal for the definition of Pls to be adopted and used across Europe. The procedure is based on a deep analysis of existing bridge management policies and available documents for inspection and evaluation existing in European countries and the main PIs used, with the objective to define a common group of quality specifications and control plans that can be assumed by all these countries.

As the focus on an efficient delivery of network performance increases, so does the interest in the relations between societal goals and PIs for both the road network and bridges or bridge elements. 
Network or even societal goals tend to be rather broad in their definition. Furthermore, there is often no exclusive relationship between PIs set at a lower level and goals at a higher level. An important notion is that in many countries, the main focus of bridge management is still the condition assessment of the particular objects or elements thereof.

The objective of COST Action TU1406 is to investigate the way bridge PIs and KPIs are collected and quantified, how PGs are specified across Europe, and finally to produce guideline documents linking collection and quantification of PIs, KPIs, PGs, standards, and practices to decision making processes. This paper shows an overview of PGs at different levels, from high-level strategic decisions to low level and system-specific requirements developed within the Action. The paper also explains how other performance aspects, like traffic safety, availability, economy, environmental and societal impacts could be quantified and used for the multi-objective bridge PGs assessment. At the end, 5 PGs and corresponding KPIs were defined: Reliability, Availability, Safety, Economy and Sustainability and based on them, Quality Control plans are defined. These, in turn, are divided into static (point in time) and dynamic (maintenance plans) control. In current practice, in most countries, the condition state or the condition index is used as a unique KPI, instead of reliability and safety.

\section{Acknowledgements}

This article is based upon the work from COST Action TU1406: Quality Specifications for Roadway Bridges. Standardization at a European Level, supported by COST (European Cooperation in Science and Technology). The financial support received by the second author from Spanish Ministry of Economy and Competitiveness rough BIA2017-86811-C2-1-R (FEDER funds) is also acknowledged

\section{References}

[1] fib. fib Model Code for Concrete Structures 2010. Wilhelm Ernst \& Sohn, 2013.

[2] Karlaftis M., Kepaptsoglou K. Performance Measurement in the Road Sector: A cross- country review of experience - Discussion Paper 2012-10 - C OECD/ITF 2012, http://www.internationaltransportforum.org/ itrc/DiscussionPapers/DP201210.pdf, 2012.

[3] Bakker J., Blom M., van den Bogaard J., Bruggink G., Dietvorst B., Klanker G., Nagtzaam G., Souw R., Vermeulen B., van der Worp J., Zwanenbeek T. Leidraad RAMS - Sturen op prestaties van systemen, Utrecht, The Netherlands: Rijkswaterstaat. 2010.

[4] Bakker J., Klatter L. Risk Based Inspection (RBI) at Rijkswaterstaat. Proceedings of the 6th international conference on Bridge Maintenance, Safety and Management, Stresa, Italy, 2012.

[5] Klanker G., Klatter L. Assessment and management of risks at bridge and network levels. Proceedings of the 7th international conference on Bridge Maintenance, Safety and Management, Shanghai, China, 2014.

[6] Rashidi, M., Lemass, B. 2011, A Decision Support Methodology for Remediation Planning of Concrete Bridges. KICEM Journal of Construction Engineering and Project Management. 2011; 1(2): 1-10.

[7] Ishizaka A., Nemery P. Multi-criteria decision analysis: methods and software. New York: John Wiley \& Sons, 2013.

[8] Keeney R. L., Raiffa H. Decisions with multiple objectives: preferences and value trade-offs. Cambridge university press, 1993.

[9] Reichert P., Schuwirth N., Langhans S. Constructing, Evaluating and Visualizing Value and Utility Functions for Decision Support. Environmental Modelling and Software, 46:283-91, 2013.

[10] Thevenot H. J., Steva E. D., Okudan G. E., and Simpson T. W. A multi-attribute utility theorybased approach to product line consolidation and selection. In ASME 2006 International Design Engineering Technical Conferences and Computers and Information in Engineering Conference: 441-450. American Society of Mechanical Engineers, 2006.

[11] Hajdin R., Kušar M., Mašović S., Linneberg P., Amado J. and Tanasić N. WG3 Technical Report - Establishment of a Quality Control Plan. 2017. 